\title{
Selection of Control Algorithms for Hybrid Electric Vehicle
}

\author{
Anosov V. N. \\ Mechatronics and automation faculty \\ Novosibirsk State Technical University \\ Novosibirsk, Russia
}

\author{
Saidov S. A. \\ Mechatronics and automation faculty \\ Novosibirsk State Technical University \\ Novosibirsk, Russia \\ st06798_1992@mail.ru
}

\author{
Yaroslavcev M. V. \\ Mechatronics and automation faculty \\ Novosibirsk State Technical University \\ Novosibirsk, Russia
}

\begin{abstract}
In the paper the main problems of power control strategies in series hybrid electric vehicles are discussed. Classical regulators are compared with neural networks, their advantages and disadvantages are shown. Experimental data sources for training neural networks and proofing results are suggested and limitations of their application scope have been considered. It is shown that improving the quality of the regulation of electric drives by using a neural network controller can lead to an increase in the reliability of regulatory systems, which subsequently leads to the saving of electricity and resources. Using the experimental data it makes possible to construction and synthesis of a neural regulator. Thus the following research directions are evaluation of optimal neural network structure and modeling its performance.
\end{abstract}

Keywords - hybrid electric vehicle, series hybrid, power installation, control strategy, neural network, fuel consumption

\section{INTRODUCTION}

Transportation in modern cities is performed mainly by cars with internal combustion engines. It is followed by emissions of greenhouse gases and spending of valuable fuels. For instance, only in Russia about 15 million tons of harmful substances are emitted per year [1]. The exhausted gases of internal combustion engines contain about 200 different components, most of which are toxic, their life cycle lasts from 2-3 minutes to 4-5 years [2-4]. It is expected that in the next $10-20$ years the internal combustion engine will remain the main energy source of road vehicles $[5,6]$.

The concept of transport type for passenger transportation is determined by the sources of energy of vehicle. One of the main directions of development of the road vehicles is application of hybrid power units combining traditional internal combustion engine with electric drive $[2,6]$. Application of hybrid electric vehicles (HEV) significantly reduces fuel consumption and greenhouse gas emission. Estimated reduction of fuel usage reaches $30-40 \%$. Another advantage of hybrid powertrain is an ability to improve dynamic properties of the vehicle increasing its acceleration. Thus, the main goals of creating a hybrid vehicle are to increase efficiency and improve the environmental situation.

The conversion of electrical energy into kinetic energy takes place on the rolling stock a part of which is spent to overcome resistance to movement, and the other part must return to the network during a regenerative braking. To determine ways for increasing the energy efficiency of electric transport, it is necessary to estimate the energy consumption for transformations of various types.

\section{Structure of Powertrain OF Hybrid Electric Vehicle}

The most effective structure of the hybrid powertrain depends on its field of application and driving cycle. Drive cycle of city road transit vehicles differs by limited maximal velocity and distance between stops with high frequency of acceleration and braking regimes interchange. In another words, the peculiarity of urban driving cycles is long-time standing in transient states. In such conditions the most suitable solution is series hybrid electric powertrain without direct mechanical link between internal combustion engine and driving wheels. Its application on urban transit rolling stock is advised in $[2,8,9]$.

In the series hybrid drive the energy of a primary energy source (PES) is transmitted to both traction drive and buffer energy storage (BES). The BES may be produced on the base of lithium-ion batteries or double electric layer capacitors.

As driving cycle is influenced by a high number of random impacts, the average power consumed from PES during each trip may substantially vary. Thus the problem of PES power management arises to keep the energy of BES in the desired limits. In the one hand, the BES should have a reserve to receive the energy generated during braking, and in the other hand it must not fall below the minimal allowed level ensuring the vehicle's ability to perform its designed dynamic properties $[2,7,9,10]$. 
Hybrid vehicle energy management strategies are discussed in the large amount of works. A number of approaches based on different principles have been proposed, including deterministic rule-based control, fuzzy logics and optimization algorithms. In its turn the optimization approach includes linear programming methods, stochastic dynamic programming, methods of game theory and genetic algorithms [2, 5-11].

To perform the investigation of energy management strategies a model of energy management system has been proposed. Its functional scheme is shown in the Fig. 1. It also possible to increase the energy efficiency of the vehicle through the development of appropriate control algorithms of the combined power plant.

\section{CAlCUlation OF Fuel CONSUMPTION AND BUfFER ENERGY STORAGE STATE OF CHARGE}

In the model driver sets its mode of operation. The setting may be presented as a dependence of traction drive power on time PTED $(t)$. The goal of energy management is finding the power of PES (a system of internal combustion engine with generator). Its dependence on time must meet the requirements of buffer energy storage level and minimum of fuel consumption. The amount of energy stored in the BES $\operatorname{EBES}(\mathrm{t})$ may be calculated by the following equation (1):

$$
\begin{aligned}
& E_{B E S}(t)=E_{0}+\int P_{I C E}(t) \cdot \eta_{I C E} P_{I C E}(t) d t- \\
& -\int P_{T M}(t) \cdot \eta_{T M} P_{T M}(t) d t
\end{aligned}
$$

where $E_{0}$ is the initial amount of BES energy;

$\eta_{\text {ICE }}$ is the total primary energy source efficiency;

$\eta_{\mathrm{TM}}$ is the efficiency of the traction drive.

In the proposed model the $\mathrm{E}_{\mathrm{BES}}(\mathrm{t})$ dependence should fulfill the following limitations. First is the condition of BES energy amount sufficiency (2). It means that in any moment of time $\mathrm{t}$

$$
\forall t: E_{B E S}(t) \in E_{\min } \ldots E_{\max }
$$

where $E_{\mathrm{MIN}}$ and $E_{\mathrm{MAX}}$ are the minimal and maximal limits of BES energy.

The second limitation is sufficiency of PES power. During the time of the trip $\mathrm{T}$ the condition (3) should be fulfilled:

$$
E_{B E S}(0)=E_{B E S}(T)
$$

The third condition is the minimum of fuel consumption. A shape of the PES power function $\mathrm{P}_{\mathrm{PES}}(\mathrm{t})$ should ensure the minimal total fuel consumption $\mathrm{G}$ during the trip time $\mathrm{T}$ (4):

$$
G=\int G^{\prime}\left(P_{I C E}(t)\right) d t
$$

where $G^{\prime}$ is specific fuel consumption per unit of energy.

The second and the third conditions must be fulfilled only on large time intervals $\mathrm{T}$, reaching $10^{3} \ldots 10^{4} \mathrm{~s}$. In the short time intervals $\mathrm{P}_{\mathrm{PES}}(\mathrm{t})$ function may not fulfill these requirements. The $\mathrm{G}\left(\mathrm{P}_{\mathrm{PES}}\right)$ dependence is determined mainly by the internal combustion engine properties. As usual each value of engine's power corresponds to optimal rotating frequency which should be maintained by control system. To improve the control quality the additional functions describing the fuel consumption in transient modes and engine start should be introduced to the model.

The minimal fuel consumption is ensured at a constant rotational speed of the internal combustion engine $[12,13]$ in various traction regimes of the vehicle, which is shown in Fig. 2. Thus the fuel consumption of the vehicle moving in the

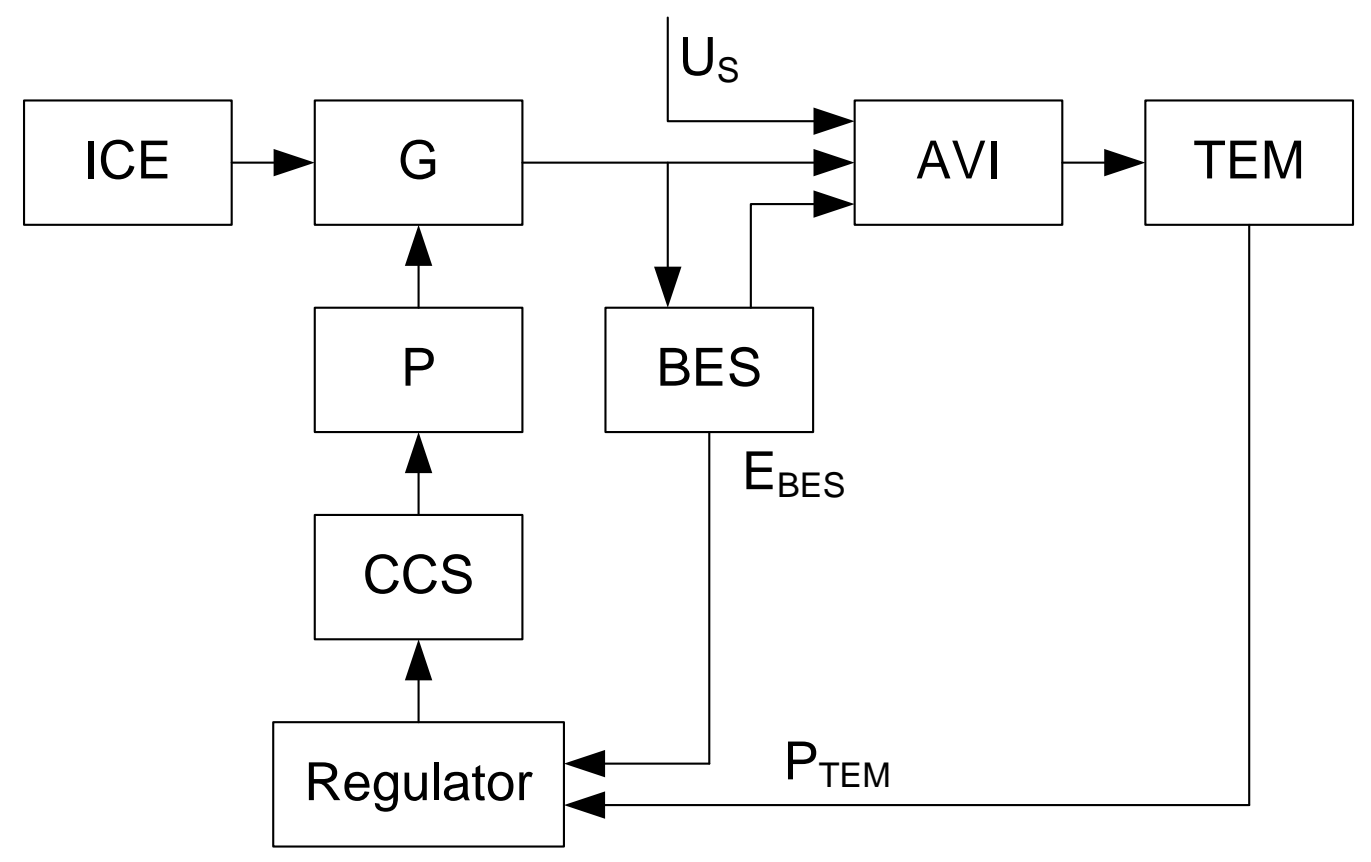

Fig. 1. Functional diagram of a series hybrid electric powertrain. 


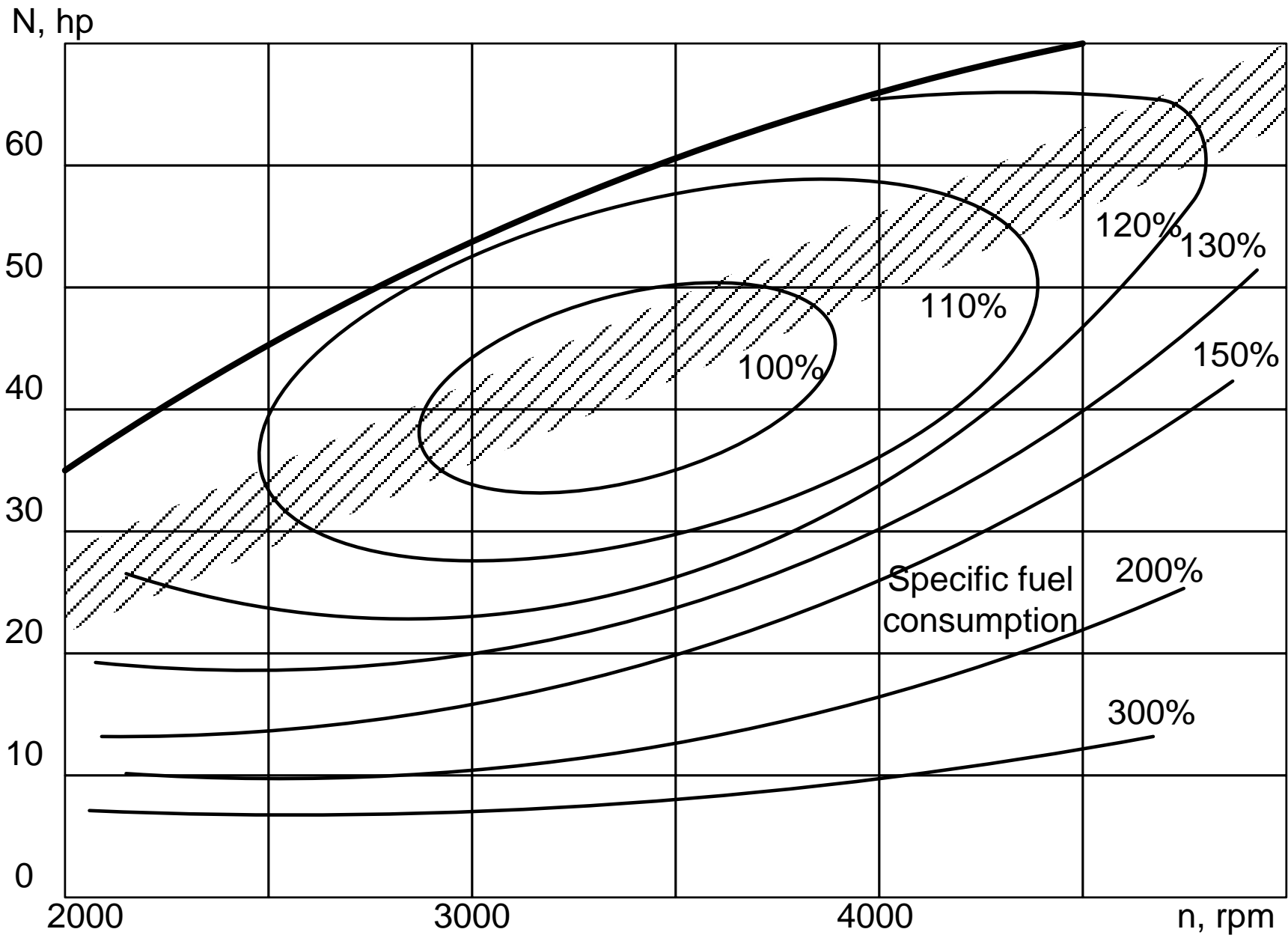

Fig. 2. Specific fuel consumption diagram of the internal combustion engine.

specified driving cycle can be calculated.

\section{Selecting THE CONTROL StRATEGY FOR THE SERIES HYBRID ELECTRIC VEHICLE}

To realize a model of hybrid drive it is needed to regulate power of ICE to prevent overcharge or full discharge of energy storage device. The simplest way to maintain a charge level is usage of standard regulators as integral (I), proportional-integral (PI), proportional-integral-differential (PID), which are widely recognized for their simplicity and high reliability in a variety of technical applications. However despite a long history of development and widespread use, classical regulators have limitations. These regulators have poor performance measures in the management of nonlinear and complex systems, as well as in case of deficiency information about the control object. Coefficients of regulators are often chosen optimally for a particular state of the object, however, when it goes to other states, these values of the coefficients no longer allow obtaining the required transient processes in terms of quality. This leads to a decrease in the quality of regulation and to an increase in energy consumption.

These problems can be solved by adapting some advanced control systems as artificial neural networks. The neural network is used to construct the regulator itself and to construct a block for tuning its coefficients. The neural network plays the role of a functional converter, which generates the regulator coefficients for each set of signals. In the construction and synthesis of neural regulator, a reference model is used that has a desired transient process of the electric drive and provides: the necessary margin of stability; the minimum static error; the necessary overshoot; the necessary time of the transient process. The error between a desired and actual transients is formed according to the structure which is shown in the Fig. 3. 
The neural network should be tuned to minimize the error between the signal received with the participation of the operator and the signal obtained during the "learning" of the neural network. The methods of controlled learning of neural networks allow to synthesize through experimental data, models of complex nonlinear control objects, and in the recognition of control objectives, neurocontrollers provide information, the possibility of their learning, generalization on the models of, control under conditions of significant nonlinearities, operation even in case of damage of individual network elements [14, 15].

Neural networks need large amount of data for training. On the base of experimental investigation of trolley bus

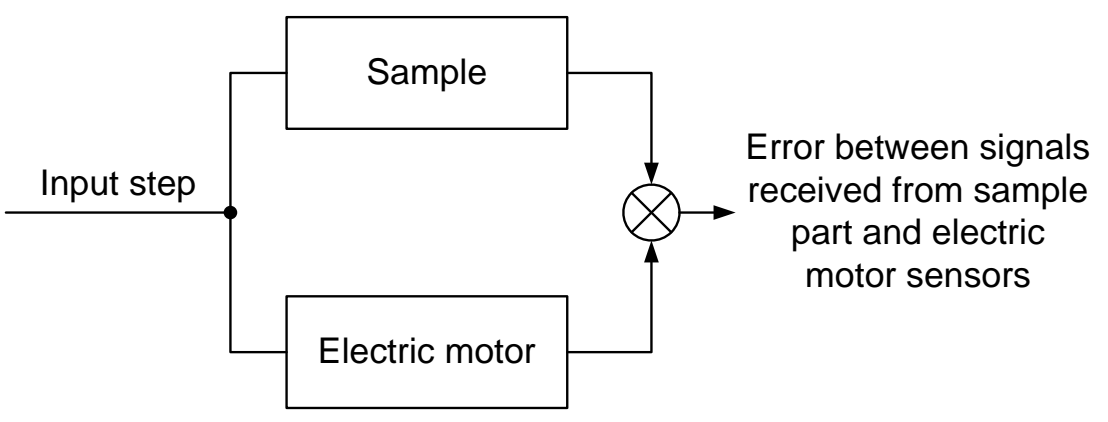

Fig. 3. Functional scheme of error calculation

\section{$\mathrm{P}, \mathrm{kW}$}

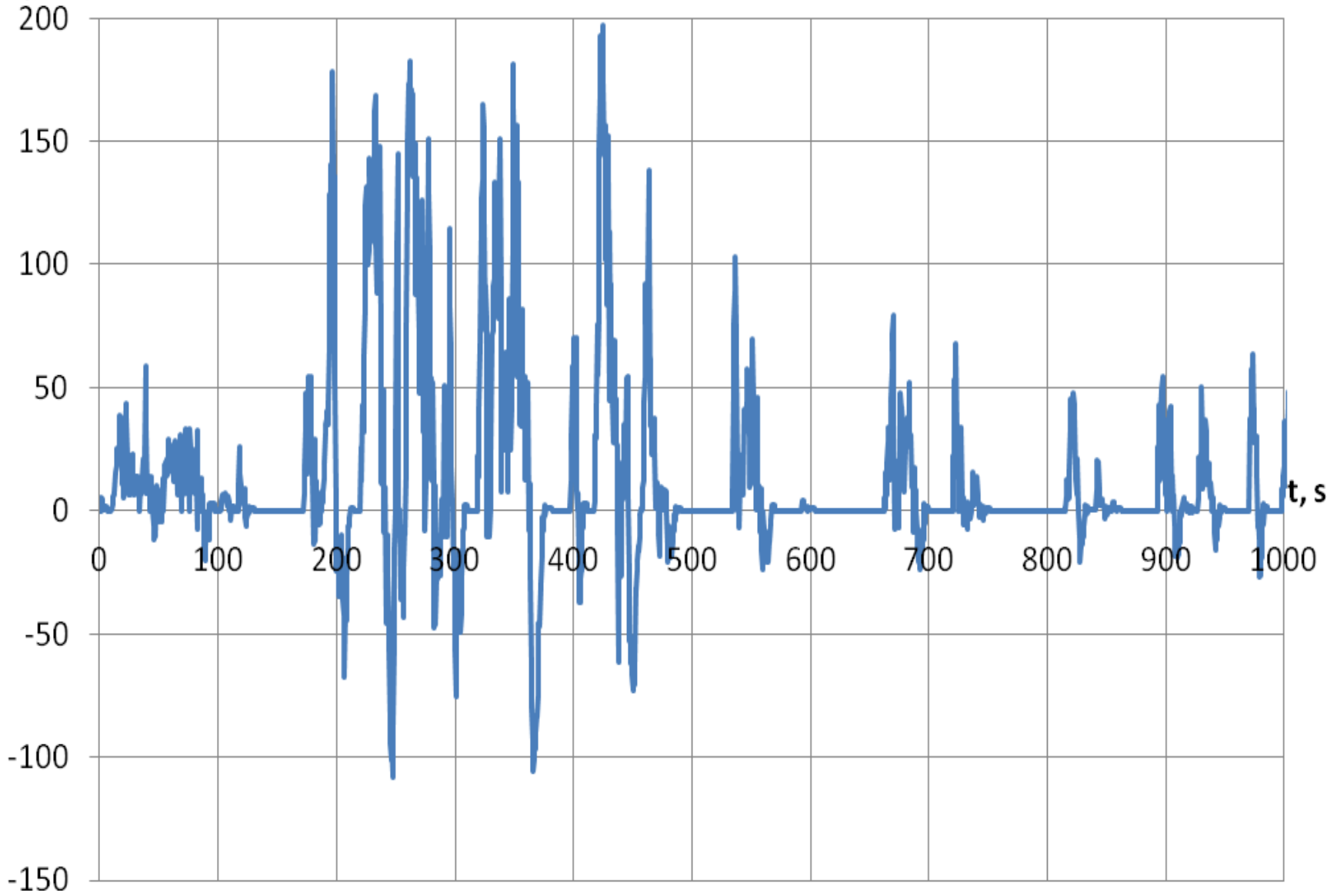

Fig. 4. An example of experimental power consumption - time dependence.

optimal behavior of the object. The advantages of neural network management in comparison with others are speed, stability, reliability, the possibility of using hidden driving cycles a traction drive power functions were obtained for about 200 hours of driving which allows effective modeling of driver's actions in different conditions $[9,16,18$ - 
21]. To analyze the results obtained with the recorder, a specialized software has been developed that allows viewing the obtained dependencies, calculating integral values characterizing the power consumption, energy consumption and analyzing the modes of the trolleybus movement in the selected section. As example in Fig. 4 a dependence of the power consumed by the traction motor obtained during the experiment is shown.

\section{SUMMARY}

The method for controlling the aggregates of a hybrid power plant with neural networks is justified. Improving the quality of the regulation of electric drives by using a neural network controller can lead to an increase in the reliability of regulatory systems, which subsequently leads to the saving of electricity and resources. Using the experimental data it makes possible to construction and synthesis of a neural regulator. Thus the following research directions are evaluation of optimal neural network structure and modeling its performance.

\section{References}

[1] State Statistic Committee of Russian Feredation, Retrieved from: http://gks.ru

[2] K. Bayindir, M. Gozukucuk, A. Teke "A comprehensive overview of hybrid electric vehicle: Powertrain configurations, powertrain control techniques and electronic control units", Energy conversion and Management, vol. 52, pp. 1305-1313, 2011.

[3] D.L. Greene, S.E. Plotkin, Reducing Greenhouse Gas Emissions from U.S. Transportation. Pew Center on Global Climate Change, 2011.

[4] R. Imam, A. Jamrah "Energy Consumption and Environmental Impacts of Bus Rapid Transit (BRT) Systems", Jordan journal of Civil engineering, vol. 6, iss. 3, pp. 328-339, 2012.

[5] M. A. Hannan, F. A. Azidin, A. Mohamed, "Hybrid electric vehicles and their challenges: A review", Renewable and Sustainable Energy Reviews vol. 29, pp. 135-150, 2014.

[6] J. V. Mierlo, G. Maggeto, P. Lataire, "Which energy source for road transport in the future? A comparison of battery, hybrid and fuel cell vehicles", Energy Conversion and Management vol. 47, iss.17, pp. 2748-2760, 2006.
[7] Siang Fui Tie,Chee Wei Tan, "A review of energy sources and energy management system in electric vehicles", Renewable and Sustainable Energy Reviews, vol. 20, pp. 82-102, 2013.

[8] R. Ghorbani, E. Bibeau, P. Zanetel, A. Karlis, "Modelling and simulation of a series parallel hybrid electric vehicle using REVS", Proceedings of the IEEE vehicle power and propulsion conference, pp. 1-6, 2008.

[9] V. N. Anosov, V. Kaveshnikov, M. V. Yaroslavtsev, "Increasing the efficiency of a traction electric drive for operation of a trolleybus", Russian Electrical Engineering, vol. 85, iss. 12, pp. 721-723, 2014.

[10] He Yiming, R. Jackeline, M. Chowdhury, P. Pisu, P. Bhavsar, "Forward power-train energy management modeling for assessing benefits of integrating predictive traffic data into plug-in-hybrid electric vehicles", Transportation Research Part D, vol. 17, pp. 201-207, 2012.

[11] R. Guizhou, M. Guoqing, Ning Cong, "Review of electrical energy storage system for vehicular applications", Renewable and Sustainable Energy Reviews, vol. 41, pp. 225-236, 2015.

[12] J. B. Heywood, Internal combustion engine fundamentals. McGraw-Hill, 1988.

[13] C. F. Taylor, Internal Combustion Engine in Theory and Practice, Second ed. Cambridge (MA): MIT Press, 1985.

[14] A. Rajagopalan, G. Washington, G. Rizzoni, Y. Guezennec, "Development of Fuzzy Logic and Neural Network Control and Advance Emissions Modeling for Parallel Hybrid Vehicles", Center for Automotive Research of The Ohio State University, Columbus, Ohio, 2003.

[15] V. V. Kruglov, M. I. Dli, R. Yu. Golunov, Fuzzy logic and artificial neural networks, Moscow: Fizmatlit, 2001.

[16] S. Khaykin, Neural networks, Moscow: Williams, 2006.

[17] A. N. Fedorov, Engineer reference book on industrial control system: design and developmen. Moscow: Infra-Inzheneriya, 2008.

[18] P. D. Wasserman, Neural computing: theory and practice. New York: Van Nostrand Reinhold Co., 1989.

[19] S. Li, Fuzzy Control. Neurocontrol and Intelligent Cybernetics. Heilongjiang, China: Haerbin University of Technology, 1998.

[20] T. Khatib, A. Mohamed, K. Sopian, M. Mahmoud, "Estimating ambient temperature for Malaysia using generalized regression neural network", International Journal of Green Energy, vol. 9, pp. 195-201, 2012.

[21] V. N. Anosov, M. V. Yaroslavtsev, "Evaluation of hybrid electric bus energy storage device capacity", proc. of 11 International forum on strategic technology (IFOST 2016) [NSTU, Novosibirsk, Russia], Pt. 2, pp. 65-67, 2016. 